\title{
Liver Biopsy Study in Diseases of Paediatric Age Group
}

\author{
Dr Dileep Kumar Jain ${ }^{1}$,Dr Malay Bajpai ${ }^{2}$,Dr Vivek Gupta ${ }^{3}$ \\ 1,3 (Departmen Of Pathology, Hind Institute Of Medical Sciences, Barabanki) \\ ${ }^{2}$ (Department Of Pathology, Rama Medical College,Hapur)
}

\begin{abstract}
To determine pattern of liver disease in the paediatric age group. Materials and Methods: In the present study of 28 cases (including 5 postmortem biopsy), liver biopsy was done, out of which 24 cases could be diagnosed histologically. Slides were prepared from paraffin-embedded blocks, stained by routine $H \& E$ and special stains and were then reviewed. The frequency of each disorder, separately and in combination with the age group or gender of the patients was calculated and compared with other similar studies. Results: Incidence of liver involvement was slightly more (58.3\%) in males. The most common age group which suffered from liver disease was 2-6 yrs (66.7\%). The commonest cause of hepatomegaly was cirrhosis of liver (57.1\%) followed by malnutrition (14.3\%), chronic venous congestion (9.5\%). Hyperbilirubinemia was present in $75 \%$ children of I.C.C., 50\% children of post hepatitis cirrhosis and 25\% of early cirrhosis. Overall it was seen in $46.7 \%$ children of cirrhosis.Liver biopsy is an essential diagnostic tool in various liver disorders, in that only in $62.6 \%$ of our cases clinical diagnosis was consistent with histopatholgical changes. Liver biopsy is an essential diagnostic tool especially in cases of unknown hepatomegaly where diagnosis can only be done after histopathological study of liver tissue.
\end{abstract}

Keywords : children, liver, histopathology

\section{Introduction}

Liver often gets involved an various diseases of pediatric age group. In many of these hepatomegaly is a primary sign while in others it is an associated finding, although liver may be diseased without any enlargement. Common diseases presenting with hepatomegaly in our set up are protein calorie malnutrition, iron deficiency anaemia, cirrhosis of liver, viral and bacterial infections and malignancies. Needle biopsy of the liver is easily done through percutaneous approach. The amount of tissue obtained, even in small infants, is usually sufficient for histologic interpretation. Percutaneous liver biopsy is safely performed in infants as young as one week of age. Needle biopsy of liver is not merely a simple, safe and inexpensive reliable histological diagnostic method in hepatic pathology but also it outlines the eiopathogensis of diseae. Now a days it is the most valuable diagnostic tool in pediatric hepatology ${ }^{[1]}$. The safety record of percutaneous liver biopsy is excellent without a single mortality and hardly any complication if done with proper care. Liver biopsies are of great importance for clinical practice because they allow assessment of the presence and severity of histopathological lesions in the liver. When added to the clinical, laboratory and imaging data, this is the basis for diagnosis, prognosis and monitoring of liver disease ${ }^{[2]}$.

Histological findings frequently help establish definitive diagnoses ${ }^{[3]}$

\section{Material And Methods}

The present observations were made on 28 children under age of $12 \mathrm{yrs}$, who were admitted in the Dept. of Pediatrics, Hind Medical College, suffering from liver diseases or in which liver involvement was suspected by the pediatrician incharge as a complication of other diseases. Following investigations with brief history and examination of the patients were carried out with special emphasis over liver biopsy.

\section{Brief History}

(a) The history of cases was documented with special reference to occurrence of jaundice, hepatomegaly, haematemesis/malena and possible etiological factors of hepatic pathology.

(b) The past history was also asked from the patient with great emphasis about any previous transfusion and jaundice.

(c) Family history of any liver disease.

\section{Laboratory Investigations}

Following laboratory investigations were carried out in every case as for as possible and wherever necessary the investigations were repeated. 
(a) Blood

(b) Urine

(c) Stool

(d) Liver Function Testes -
$\mathrm{Hb} \%$, TLC, DLC

Platelet count

(Dacie and Lewis, 1984)

General blood picture

Bleeding time (Ivy's method)

Clotting time (Capillary method)

Mid stream morning sample was collected for-

Routine/Microscopic examination.

Bile pigments (Fouchet's test).

Bile salts (Hay's test) - using sulphur powder.

Urobilinogen (Ehrlich's aldehyde test).

For ova and cysts.

Occult blood (wherever required).

$5 \mathrm{ml}$. of blood was collected in plain sterile vial and serum was separated and following estimations were done :

1. Serum bilirubin

2. S.G.P.T. Serum alkaline phosphatase

3. Total and differential serum proteins by Biuret method

○ (e) Biopsy of liver was done by using Vim silverman's needle.

(f) Histopathological examination of biopsy tissue.

\section{Histology Of The Tissue -}

The tissue obtained from the biopsy was kept in $10 \%$ buffered formal saline. After dehydration and processing paraffin blocks were made and the sections were cut at 4 to $5 \mu$ thickness and stained with haematoxyline and eosin ${ }^{[4]}$

Following special stains were also used to establish the diagnosis.

i. Reticulin stain

ii. Modified Perls' Prussian Blue reaction for iron.

iii. Rhodanine stain for copper

iv. Van Gieson stain

\section{Observations}

In the present study of 28 cases (including 5 postmortem biopsy), liver biopsy was done, out of which 24 cases could be diagnosed histologically. In 2 cases no liver tissue could be obtained. In remaining 2 cases tissue was too small for the diagnosis.

Table - I

Showing distribution of cases according to clinical diagnosis in present study.

\begin{tabular}{llcc}
\hline S. No. & Clinical diagnosis & No. of cases & Percentage \\
\hline l. & Cirhosis of liver & 8 & $28.57 \%$ \\
2. & Infectious hepatitis & 3 & $10.71 \%$ \\
3. & Malnutrition & 3 & $10.71 \%$ \\
4. & Hepatic Encephalopathy & 3 & $10.71 \%$ \\
5. & Unknown hepatomegaly & 3 & $10.71 \%$ \\
6. & Miliary Tuberculosis & 1 & $3.57 \%$ \\
7. & Tubercular Meningitis with pyopericardium & 1 & $3.57 \%$ \\
8. & Aplastic anaemia with Haemosidesosis & 1 & $3.57 \%$ \\
9. & Tropical splenomegaly Syndrome & 1 & $3.57 \%$ \\
10. & Reye's Syndrome & 1 & $3.57 \%$ \\
11. & Congenital infection & 1 & $3.57 \%$ \\
12. & Neonatal Septicaemia & 1 & $3.57 \%$ \\
13. & Primary Carcinoma of liver & 1 & $3.57 \%$ \\
& & & \\
\hline & TOTAL & & \\
& & & $\mathbf{1 0 0 . 0 0 \%}$ \\
\hline
\end{tabular}


Out of 24 cases of liver disease largest number 15 cases (62.6\%) was that of cirrhosis followed by fatty change. $3(12.5 \%)$ and chronic venous congestion $2(8.3 \%)$ cases.

\section{Discussion}

The present study was aimed to establish etiopathogenisis and diagnosis of various liver diseases and those diseases where liver in subsequently involved in pediatric age group by needle biopsy of liver. In the present study, liver biopsy was performed in 28 children under the age of 12 year. Tissue obtained was adequate in $85.7 \%$ cases while inadequate in $7.15 \%$ and complete failure in $7.15 \%$ cases. On examination hepatomegaly was the most common finding (87.5\%). It was seen in all the cases of early cirrhosis, fatty change, chronic venous congestion. 83\% cases of post hepatitis cirrhosis, $75 \%$ cases of Indian childhood cirrhosis. It was followed by anaemia (83.3\%), jaundice (29.5\%), splenomegaly $(28.3 \%)$ and ascites (17\%). Jaundice was a feature in $34 \%$ cases of post hepatitis cirrhosis, $25 \%$ cases of early cirrhosis $50 \%$ cases of I.C.C. and single case of early intrahepatic biliary cirrhosis and infectous hepatitis. These signs and symptoms have also been described by most of worker in this field

We have oobserved that all the patients of I.C.C. were below 4 yrs of age (most common age distribution 6 month -4 yrs). Of this 3 cases presented between 2-4 yrs. Post hepatitis cirrhosis was seen more in male (83.3\%). Robbins and Kumar reported that about $1 / 2$ th cases of post necrotic cirrhosis follow long standing chronic active hepatitis B-infection but majority has no cause for post necrotic cirrhosis. All the children of fatty liver were between 2-6 yrs. and underweight for their age. Fatty change were commoner in males as compared to females (Male : Female 2:1). They all were from lower socio-economic status. Chronic active hepatitis was diagnosed in a child having paste history of jaundice. According to Robbins and Kumar (30\% of individual who develop chronic active viral hepatitis have had an obvious preceding acute attack of icteric hepatitis. Infectious hepatitis was diagnosed in an eleven year old girl presented with jaundice. According to Satya Gupta, infectious hepatitis is the commonest cause of jaundice in children. He also observed that it is commonest in pre-school age. Tuberculosis of liver was seen in a ten month old female infant having military tuberculosis presented with low grade fever, failure to thrive, hepato splenomegaly. Haemosiderosis and early intrahepatic biliary cirrhosis was seen in males. In early cirrhosis and chronic venous congestion, sex ratio was equal. The overall male to female ratio for liver diseases was 1.4:1. Our findings are consistent with findings of Kanase et al.. In this respect no obvious reason could be given for male predominance. In tuberculous granulomatous hepatitis liver function tests were within normal limit in our study. In fatty charge of liver, $66.7 \%$ children showed hypoproteinemia. Albumin was reduced in all the cases. Hyperglobulinemia was seen in 66.7 $\%$ cases. A/G ratio reversed in all.). One child suffering from chronic active hepatitis had moderately increased SGPT and markedly increased alkaline phosphatase, rest of the liver functions were normal.

\section{Haematological Changes}

Leucopenia and thrombocytopenia was seen in $20 \%$ cases of cirrhosis. It could be due to associated hypersplenism. ${ }^{8]}$ PBS revealed microcytic hypochromic anaemia in $60.0 \%$ cases, dimorphic picture in $20.0 \%$ and normocytic normochromic in $13.3 \%$ cases of cirrhosis. Our findings are in accordance with findings of Jhon Macleod ${ }^{[9]}$. Anaemia in these cases may be due to nutritional deficiency or chronic blood loss from gut or both. $66.7 \%$ children of fatty change liver revealed iron deficiency anaemia and in $33.3 \%$ dimorophic anaemia was seen due to nutritional deficiency.

Bleeding and clotting time of all the patients were within normal limit. It may be stressed again that liver biopsy is an essential diagnostic tool in various liver disorders in that in $62.2 \%$ of our cases clinical diagnosis was consistent with histopahtological changes. Baggentoss reviewed 600 liver biopsy cases in relation to clinical diagnosis and in $70 \%$ cases, a correct and accurate comparison could be made. ${ }^{[1]}$ Gupta et al. reported that in 55\% of cases clinical diagnosis was consistent with histological diagnosis in an experience with 256 liver biopsy in children, simultaneously liver biopsy helped in establishing aetiopathogenesis of the diseases. ${ }^{[6]}$

\section{Conclusion}

The present study of 28 children (under the age of $12 \mathrm{yrs}$.) of various liver pathology who were subjected to clinical, haematological, biochemical examination yielded the following results - Incidence of liver involvement was slightly more $(58.3 \%)$ in males.

1. The most common age group which suffered from liver disease was $2-6$ yrs $(66.7 \%)$.

2. The commonest presenting feature in our children were fever, anorexia, pain in abdomen, jaundice.

3. On examination hepatomegaly was the commonest presenting sign $(87.5 \%)$, followed by anaemia (83.3\%), jaundice $(29.5 \%)$, splenomegaly $(28.3 \%)$ and ascites (17\%).

4. The commonest cause of hepatomegaly was cirrhosis of liver (57.1\%) followed by malnutrition (14.3\%), chronic venous congestion (9.5\%). 
5. Hyperbilirubinemia was present in $75 \%$ children of I.C.C., $50 \%$ children of post hepatitis cirrhosis and $25 \%$ of early cirrhosis. Overall it was seen in $46.7 \%$ children of cirrhosis.

6. S.G.P.T. was elevated in $75 \%$ cases of I.C.C. $33.3 \%$ of post hepatitis cirrhosis. $25 \%$ cases of early cirrhosis. Overall it was raised in $46.7 \%$ cases of cirrhosis.

7. Elevated levels of alkaline phosphatase were found in $75 \%$ cases of early cirrhosis, $75 \%$ cases of I.C.C. and $50 \%$ cases of post hepatitis cirrhosis. Rise in I.C.C. was of sever grade. Overall it was raised in $66.7 \%$ cases of cirrhosis of liver.

8. A/G ratio reversed in all the cases of I.C.C., $66.7 \%$ cases of post hepatitis cirrhosis, $75 \%$ cases of early cirrhosis. Overall A/G ratio reversed in $73.3 \%$ cases of cirrhosis. It also reversed in fatty liver, infective hepatitis, haemosiderosis.

9. Liver biopsy is an essential diagnostic tool in various liver disorders, in that only in $62.6 \%$ of our cases clinical diagnosis was consistent with histopatholgical changes.

10. Presence of copper in liver was detected in $75 \%$ cases of Indian childhood cirrhosis indicating the importance of copper in aetio-pathogensis of I.C.C.

11. Excessive deposition of iron was detected in a case of aplastic anaemia with hepatomegaly pointing that cause of hepatomegaly was haemosiderosis confirmed by Perls' stain.

12. Post hepatitis cirrhosis was the commonest (40\%) type of cirrhosis seen in our study followed by Indian childhood cirrhosis $(26.7 \%)$.

13. Hepatomegaly due to tuberculosis was confirmed only by liver biopsy.

14. Liver biopsy is an essential diagnostic tool especially in cases of unknown hepatomegaly where diagnosis can only be done after histopathological study of liver tissue.

\section{References}

[1]. Behraman, E. Richard \& Vaughan, C. Victor : Nelson Text book of pediatrics. $13^{\text {th }}$ ed., W.B. Saunders Company. 1987.

[2]. Ridaura-Sanz C. La biopsia en el diagnóstico de la enfermedad pediátrica. Acta Pediatr Mex 2008;29(4):216-26.

[3]. Rastogi A, Krishnani N, Yachha SK, Khanna V, Poddar U, Lal R. Histopathological features and accuracy for diagnosing biliary atresia by prelaparotomy liver biopsy in developing countries. J Gastroenterol Hepatol 2009;24(1):97-102

[4]. Bancroft, J.D. \& Stevens, A., (ed) : Theroy and practice of histological technique : Churchill livingstone, Edinberg. 1982

[5]. Robbins, L. Stanley and Kmuar Vinay : Basic Pathology $4^{\text {th }}$ ed., W.B. Saunders Company, 1987.

[6]. Gupta, S., Puri. R.K. and Balasubrahmanyam, M. : Needle biopsy of the liver in pediatric practice. Ind. J. Pediat. $1970,37: 565$

[7]. Kanase. B.A., Deodhar, K.P., Kandoth. W.K. : Indications for liver biopsy in India. Ind. Pediat., 1981, 48 : 589Bhakoo, O.N., Wallia. B.N.S. - Ind. Pediat, 1981, 48: 393.

[8]. Bhagwat, A.G. \& Wallia. B.N.S - Ind. Pediat, 1981, $48: 393$.

[9]. Macleod. John : Davidson's Principles and practice of medicine. $14^{\text {th }}$ ed., Churchill livingstone, 1984

[10]. Bhave. S.A., sindhaye, D.G., Pandit, A.N. \& Tanner, M.S - Ind. Pediat., 1983, 20 : 741.

[11]. Baggentoss, A.H. : Post-hepatitic and alcoholic cirrhosis. Gasteroenterology, 1952, 22 : 157.

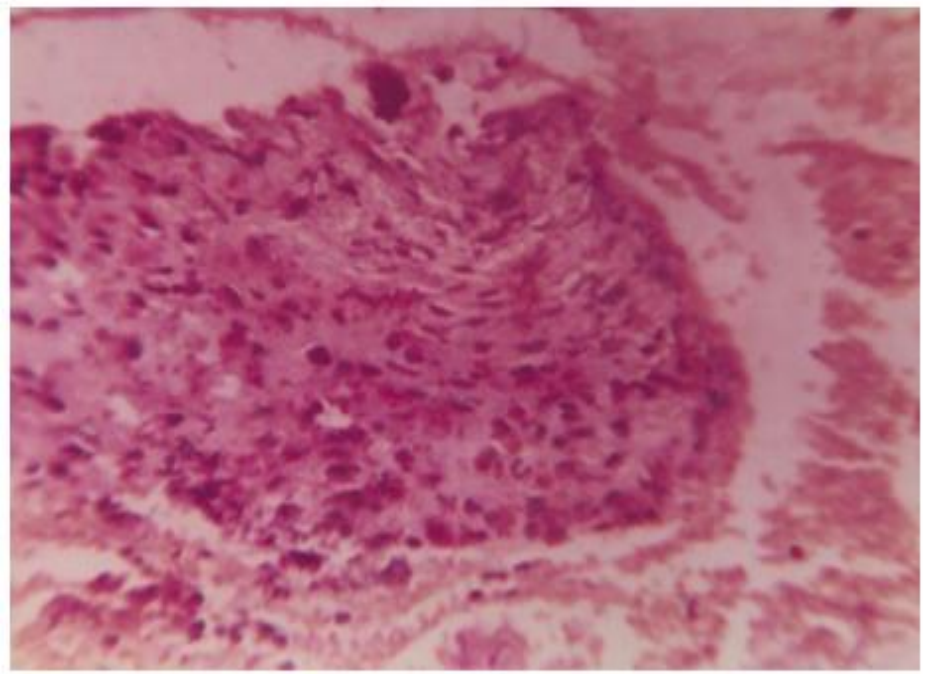

Photo micrograph of Liver Biopsy tissue showing marked deposition of copper in liver - Reddish granules $(3+)$

I.C.C. - Rhodanine stain $\times 280$ 


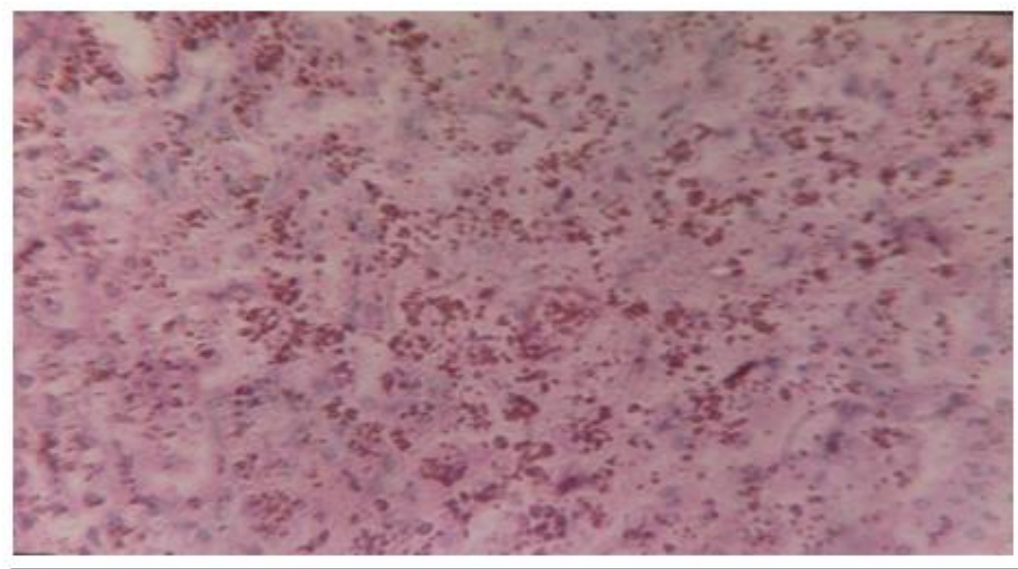

Photo micrograph of Liver Biopsy tissue showing extensive deposition of haemosiderin.

Haemosiderosis-H \& E-Haemosiderin (Golden brown).

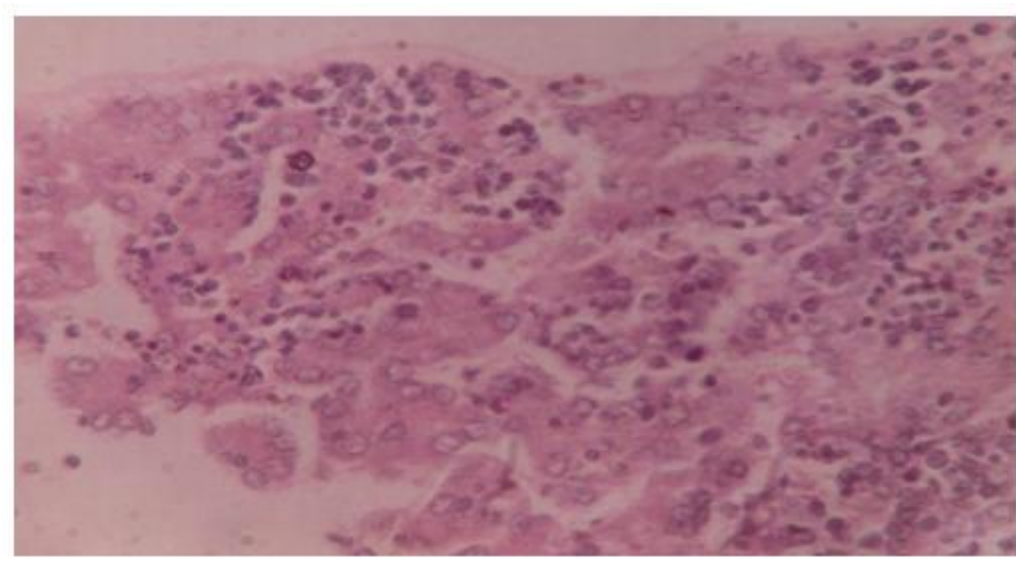

\section{Chronic venous congestion -}

Distended sinusoids with evidence of extrameddulary haemopoisis-H \& E stain $\times 280$

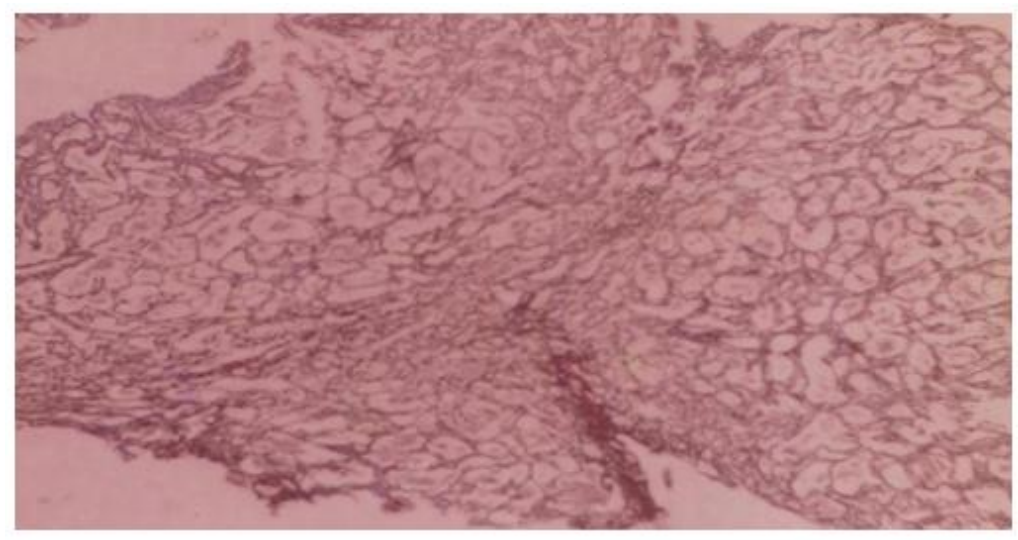

Photo micrograph of Liver Biopsy tissue showing fibrosis around group of hepatocytes as well as around individual hepatocytes - I.C.C. Retculin $\times 70$ 